\title{
Down-regulation of long noncoding RNA RP11-713B9.1 contributes to the cell viability in non-small cell lung cancer (NSCLC)
}

\author{
JUN CAI $^{1 *}$, XUAN WANG $^{1 *}$, HU HUANG $^{2 *}$, MENG WANG $^{1}$, ZHIHONG ZHANG $^{3}$, \\ YAN HU $^{1}$, SHIHUA YU ${ }^{1}$, YONGHUA YANG ${ }^{1}$ and JIYUAN YANG ${ }^{1}$ \\ ${ }^{1}$ Department of Oncology, First Affiliated Hospital of Yangtze University, Jingzhou, Hubei 434000; \\ ${ }^{2}$ Department of Oncology, The 161th Hospital of PLA, Jiang'an, Wuhan, Hubei 430010; \\ ${ }^{3}$ Department ofOncology, Gong'an County People's Hospital, Jingzhou, Hubei 434000, P.R. China
}

Received January 13, 2017; Accepted July 6, 2017

DOI: $10.3892 / \mathrm{mmr} .2017 .7026$

\begin{abstract}
Early diagnosis is essential to reduce lung cancer-associated morbidity and mortality rates; however the lack of diagnostic biomarkers for lung cancer has made this difficult. Recent studies have demonstrated that long noncoding RNAs (lncRNAs) serve important roles in cancer occurrence and progression. The present study investigated the novel lncRNA RP11-713B9.1, which is the antisense transcript of tumor suppressor in lung cancer 1 (TSLC1). The expression levels of RP11-713B9.1 and TSLC1 in non-small cell lung cancer were determined using reverse-transcription quantitative polymerase chain reaction, which revealed that the expression of RP11-713B9.1 and TSLC1 was significantly downregulated in tumor tissue compared with that in adjacent normal tissue samples. In addition, the expression of RP11-713B9.1 was identified to be positively correlated with the expression of tumor suppressors TSLC1, CYLD lysine 63 deubiquitinase and APC WNT signaling pathway regulator, and negatively correlated with B-Raf proto-oncogene serine/threonine kinase expression. Furthermore, the overexpression of RP11-713B9.1 resulted in significant upregulation of TSLC1 and inhibition of H460 cell viability, while the opposite effects were observed following the knockdown of RP11-713B9.1 in A549 cells. Taken together, the results of the current study suggest that lncRNA RP11-713B9.1 serves as a promising biomarker and potential therapeutic target for non-small cell lung cancer.
\end{abstract}

Correspondence to: Dr Jiyuan Yang, Department of Oncology, First Affiliated Hospital of Yangtze University, 8 Hangkong Road, Jingzhou, Hubei 434000, P.R. China

E-mail:scvtli@163.com

${ }^{*}$ Contributed equally

Key words: non-small cell lung cancer, long non-coding RNA, TSLC1, lncRNA RP11-713B9.1

\section{Introduction}

Lung cancer is one of the most common cancers worldwide and the first leading cause of the cancer-related death $(1,2)$. Despite tremendous efforts made to improve lung cancer treatment including surgery, chemotherapy and radiotherapy, a substantial number of patients still face the high risk of drug resistance and subsequent tumor metastasis (3-5). Moreover, metastasis is the major contributor to morbidity and mortality in patients with non-small cell lung cancer (NSCLC). Therefore, further research into the potential molecular basis of NSCLC progression is pivotal to improving the treatment and prognosis of NSCLC.

Long noncoding RNAs (lncRNAs) are an important class of the noncoding RNA family, longer than 200 nucleotides without evident protein-coding capabilities (6). Increasing evidence has showed that IncRNAs act a crucial role in cancer occurrence and progression (7-10). Some lncRNAs can participate in diverse biological processes, including cell viability, modulation of migration and invasion (11-13). However, compared to the well-characterized microRNA, the functions of lncRNAs have not been fully unravelled.

Recent studies have identified that IncRNAs are involved in NSCLC pathogenesis $(14,15)$, which provide a new insight into the clinical benefit in NSCLC treatment. TSLC1 (also known as CADM1, IGSF4, SynCAM, SgIGSF and Necl-2) (16-18) has been proved as a tumor suppressor gene in various cancers including nasopharyngeal cancer, breast cancer, gastric cancer, pancreatic cancer, colorectal cancer, cervical cancer and prostate cancer (19-25). Intriguingly, recent study has showed that TSLC1 is significantly down-regulated in NSCLC tissues and cell lines, and elevated expression of TSLC1 inhibits NSCLC cell viability, migration, and invasion (26). BRAF is well known as a genetic mutation that could activate oncogenes, which have been identified to be associated with NSCLC (27). CYLD and APC are the negative regulators of Wnt signaling pathway and also reported to be associated with $\operatorname{NSCLC}(28,29)$. Nonetheless, as the antisense RNA of TSLC1 (30), lncRNA RP11-713B9.1 expression and its biological role in NSCLC development and progression remain largely unknown. 
In our study, we discovered that the expression of RP11-713B9.1 in NSCLC samples was significantly lower than that in the adjacent normal lung tissues. The expression of RP11-713B9.1 and TSLC1 was positively correlated and co-regulated in NSCLC. Furthermore, overexpression and knockdown experiments were performed to explore the role of RP11-713B9.1 in NSCLC cells. We found that RP11-713B9.1 overexpression inhibited the NSCLC cells viability, whereas RP11-713B9.1 knockdown showed the opposite results. On the basis of our findings, we speculate that lncRNA RP11-713B9.1 may serve as a novel potential biomarker in NSCLC treatment.

\section{Materials and methods}

Lung cancer samples. Samples of surgically removed tumors used in this study were collected from 46 patients with a postoperative diagnosis of NSCLC from 2010 to 2012 in First Affiliated Hospital of Yangtz University. Demographic and clinical characteristics of 46 NSCLC patients were shown in Table I. No patients had received therapy before surgery. Each participated patient signed a written informed consent, which was in accordance with our institutional ethical guidelines. The utilization of tumor tissues for this study was all approved by the ethical committee of First Affiliated Hospital of Yangtz University. The 46 NSCLC tumor samples were quick frozen at the time of resection until analysis.

Cancer cell lines. The human normal lung epithelial cell BEAS-2B, the commercial human NSCLC cell lines H460, H1975, A549 were obtained from the American Tissue Culture Collection (ATCC, Manassas, VA, USA), and cell lines were maintained in Dulbecco's modified Eagle's medium (DMEM) medium (Gibco, Carlsbad, CA, USA) supplemented with $10 \%$ fetal bovine serum (FBS) (Invitrogen, Carlsbad, $\mathrm{CA}, \mathrm{USA})$ in a humidified atmosphere of $5 \%(\mathrm{v} / \mathrm{v}) \mathrm{CO}_{2}$ and $95 \%$ air at $37^{\circ} \mathrm{C}$.

Real-Time Quantitative PCR. Total RNA was extracted from the NSCLC tumor tissue and adjacent normal tissue using the Trizol Total RNA Reagent (Invitrogen, Carlsbad CA, USA) following the manufacturer's protocol. cDNA synthesis was performed with $2 \mu \mathrm{g}$ total RNA using the RevertAidTM H Minus First Strand cDNA Synthesis Kit (Takara, Ohtsu, Japan). The primers were obtained from GenePharma (Shanghai, China) and the sequences were shown in Table II. Real-Time Quantitative PCR was performed using the SYBR PrimeScript Real-Time Quantitative PCR kit (Takara, Ohtsu, Japan) in an Applied Biosystems 7500 Fluorescent Quantitative PCR System (Applied Biosystems, Foster City, CA, USA). The reaction mixtures started at $95^{\circ} \mathrm{C}$ for $30 \mathrm{sec}$, followed by 40 amplification cycles of $95^{\circ} \mathrm{C}$ for $5 \mathrm{sec}$ and $60^{\circ} \mathrm{C}$ for $34 \mathrm{sec}$. The quantification of gene expression was performed using the $\Delta \Delta \mathrm{CT}$ calculation with $\mathrm{CT}$ as the threshold cycle.

Overexpression of RP11-713B9.1 in A549 cell line. pcDNA-RP11-713B9.1 was cloned into BamHI-EcoRI sites of pcDNA3.1. The RP11-713B9.1 low-expressing A549 cells were transfected with pcDNA-RP11-713B9.1 using Lipofectamine 2000 (Invitrogen, US) according to the manufacturer's instructions. Cells were collected after transfection for RNA extraction, MTT cell viability assay.

Knockdown of RP11-713B9.1 in H460 cell line. For small interfering RNA (siRNA) analysis, siRNA for the RP11-713B9.1 sequence and negative-control (NC) siRNA were given from GenePharma (Shanghai, China). The target sequences of RP11-713B9.1 siRNA were shown in Table III, which were also used in our previous literature (30). H460 cells were plated to the 12 -well plates with a density of $2 \times 10^{3}$ cells per well and cultured at least $24 \mathrm{~h}$ before transfection. siRNA transfection was performed with X-tremeGENE transfection reagent (Roche) according to the manufacturer's instructions. High-expressing RP11-713B9.1 in H460 cell line was harvested or fixed $48 \mathrm{~h}$ after transfection for RNA extraction, MTT assay.

Cell viability assay. After transfection, cell viability was tested using a 3-(4,5-Dimethyl-thiazol-2-yl)-2,5-diphen yltetrazolium bromide (MTT) assay (Promega) according to the manufacturer's instruction. A549 and H460 cells were seeded at a density of $2 \times 10^{3}$ cells per well in 96-well plates and maintained in Dulbecco's modified Eagle's medium (DMEM) supplemented with $10 \%$ FBS for 24-68 h prior to transfection with siRNA. Cells were transfected using 25 nM RP11-713B9.1 siRNA or control NC siRNA in serum-free and antibiotic-free DMEM. After transfection 3-5 days, cells were analyzed using the MTT reagent for another $4 \mathrm{~h}$. $100 \mu \mathrm{l}$ DMSO was added to dissolve the MTT-formazan formed by metabolically viable cells and then shaken for homogeneity. Optical density was measured at 24, 48 and $72 \mathrm{~h}$ after transfection on a microplate reader with the wave length of $490 \mathrm{~nm}$.

Statistical analyses. Differences between two groups were carried out using Student's t-test. One-way ANOVA was used for the comparison of multiple groups $(>2)$. The data variables were shown as the mean \pm SD. Correlation between genes expression was analyzed using Pearson's correlation. Statistical analyses were conducted using SPSS version 18.0 (SPSS, Chicago, IL, USA). For all statistical analyses, ${ }^{*} \mathrm{P}<0.05$ was considered statistically significant.

\section{Results}

Both RP11-713B9.1 and TSLC1 were down-regulated in the NSCLC tissue samples. The RP11-713B9.1 and TSLC1 expression levels were assessed through paired specimen obtained from 46 patients with lung cancer. And each tumor sample was normalized to the relative adjacent non-tumor tissue. The data showed that both RP11-713B9.1 and TSLC1 expression were significantly down-regulated in the NSCLC tissues $(\mathrm{P}<0.001)$ (Fig. 1A, B).

Expression of RP11-713B9.1 was correlated with TSLC1 and other tumor related genes. The correlation of RP11-713B9.1 expression with TSLC1 was assessed using Real-Time Quantitative PCR. The data suggested that RP11-713B9.1 expression was positively correlated with TSLC1 expression $(\mathrm{R}=0.62, \mathrm{P}<0.0001$, Fig. 2A). In addition, the connections between RP11-713B9.1 expression and other tumor regulating 
Table I. Demographic and clinical characteristics of 46 NSCLC patients.

\begin{tabular}{|c|c|c|c|c|c|}
\hline Variables & $\begin{array}{c}\text { NSCLC patients } \\
\mathrm{n}(\%)\end{array}$ & $\begin{array}{c}\text { Deaths } \\
\mathrm{n}=22\end{array}$ & $\begin{array}{c}\text { MST } \\
\text { (months) }\end{array}$ & $\begin{array}{c}\text { Log-rank } \\
\text { P }\end{array}$ & $\begin{array}{l}\text { Crude HR } \\
(95 \% \mathrm{CI})\end{array}$ \\
\hline \multicolumn{6}{|l|}{ Age } \\
\hline$\leq 65$ & $21(0.46)$ & 10 & 25.9 & - & 1.00 (Reference) \\
\hline$>65$ & $25(0.54)$ & 12 & 24.3 & 0.83 & $1.093(0.48-2.49)$ \\
\hline \multicolumn{6}{|l|}{ Sex } \\
\hline Male & $40(0.87)$ & 13 & 30.7 & - & 1.00 (Reference) \\
\hline Female & $6(0.13)$ & 9 & 24.9 & 0.13 & $0.52(0.22-1.22)$ \\
\hline \multicolumn{6}{|l|}{ Smoking } \\
\hline Never & $7(0.15)$ & 2 & 40.1 & - & 1.00 (Reference) \\
\hline Ever & $39(0.85)$ & 20 & 31.7 & $<0.01$ & $14.26(3.30-61.64)$ \\
\hline \multicolumn{6}{|l|}{ Histology } \\
\hline Adenocarcinoma & $21(0.46)$ & 9 & 25.3 & - & 1.00 (Reference) \\
\hline Squamous cell carcinoma & $15(0.33)$ & 8 & 21.9 & 0.39 & $1.52(0.59-3.95)$ \\
\hline Others & $10(0.21)$ & 5 & 17.6 & 0.66 & $1.28(0.43-3.83)$ \\
\hline \multicolumn{6}{|l|}{ Clinical stage } \\
\hline Early & $12(0.26)$ & 4 & 58.4 & - & 1.00 (Reference) \\
\hline Advanced & $34(0.74)$ & 18 & 12.8 & 0.003 & $5.36(1.80-15.93)$ \\
\hline \multicolumn{6}{|l|}{ Surgery } \\
\hline No & $29(0.63)$ & 14 & 22.7 & - & 1.00 (Reference) \\
\hline Yes & $17(0.37)$ & 8 & 32.1 & 0.004 & $0.19(0.06-0.59)$ \\
\hline \multicolumn{6}{|l|}{ Chemotherapy } \\
\hline No & $11(0.24)$ & 10 & 23.9 & - & 1.00 (Reference) \\
\hline Yes & $35(0.76)$ & 12 & 25.2 & 0.98 & $1.01(0.44-2.34)$ \\
\hline \multicolumn{6}{|l|}{ Radiotherapy } \\
\hline No & $25(0.54)$ & 11 & 20.4 & - & 1.00 (Reference) \\
\hline Yes & $21(0.46)$ & 11 & 22.9 & 0.58 & $0.79(0.34-1.83)$ \\
\hline
\end{tabular}

Table II. Primers Real-Time Quantitative PCR analysis.

\begin{tabular}{lll}
\hline Gene name & \multicolumn{1}{c}{ Forward } & \multicolumn{1}{c}{ Reverse } \\
\hline$\beta$-actin & 5'-CCACTGGCATCGTGATGGA-3' & 5'-CGCTCGGTGAGGATCTTCAT-3' \\
RP11-713B9.1 & 5'-TGACAAAGGCAGGAGGTA-3' & 5'-GCACTATGGCTGAGGAAA-3' \\
TSLC1 & 5'-ATGGCGAGTGTAGTGCTGC-3' & 5'-GATCACTGTCACGTCTTTCGT-3' \\
CYLD & 5'-TTCACTGACGGGGTGTACCA-3' & 5'-CAGGACCTGCGTAATCACTTTC-3' \\
APC & 5'-TCCTCCGCAATGTGTCCAG-3' & 5'-AGGCTGTGCGAAGTCAGATG-3' \\
BRAF & 5'-AATACACCAGCAAGCTAGATGC-3' & 5'-AATCAGTTCCGTTCCCCAGAG-3' \\
\hline
\end{tabular}

Table III. siRNA for RP11-713B9.1 sequences (30).

\begin{tabular}{ll}
\hline Name & \multicolumn{1}{c}{ The sequences } \\
\hline RP11-713B9.1-si1 & Sense strand 5'-rGrUrArCrCrUrCrCrUrGrCrCrUrUrUrGrUrCrArArGrCrCAA-3' \\
& Antisense strand 5'-rUrUrGrGrCrUrUrGrArCrArArArGrGrCrArGrGrArGrGrUrArCrArA-3' \\
RP11-713B9.1-si2 & Sense strand 5'-rGrArCrCrUrArUrCrGrArGrArArCrUrGrArGrArGrCrGrACA-3' \\
& Antisense strand 5'-rUrGrUrCrGrCrUrCrUrCrArGrUrUrCrUrCrGrArUrArGrGrUrCrArG-3'
\end{tabular}



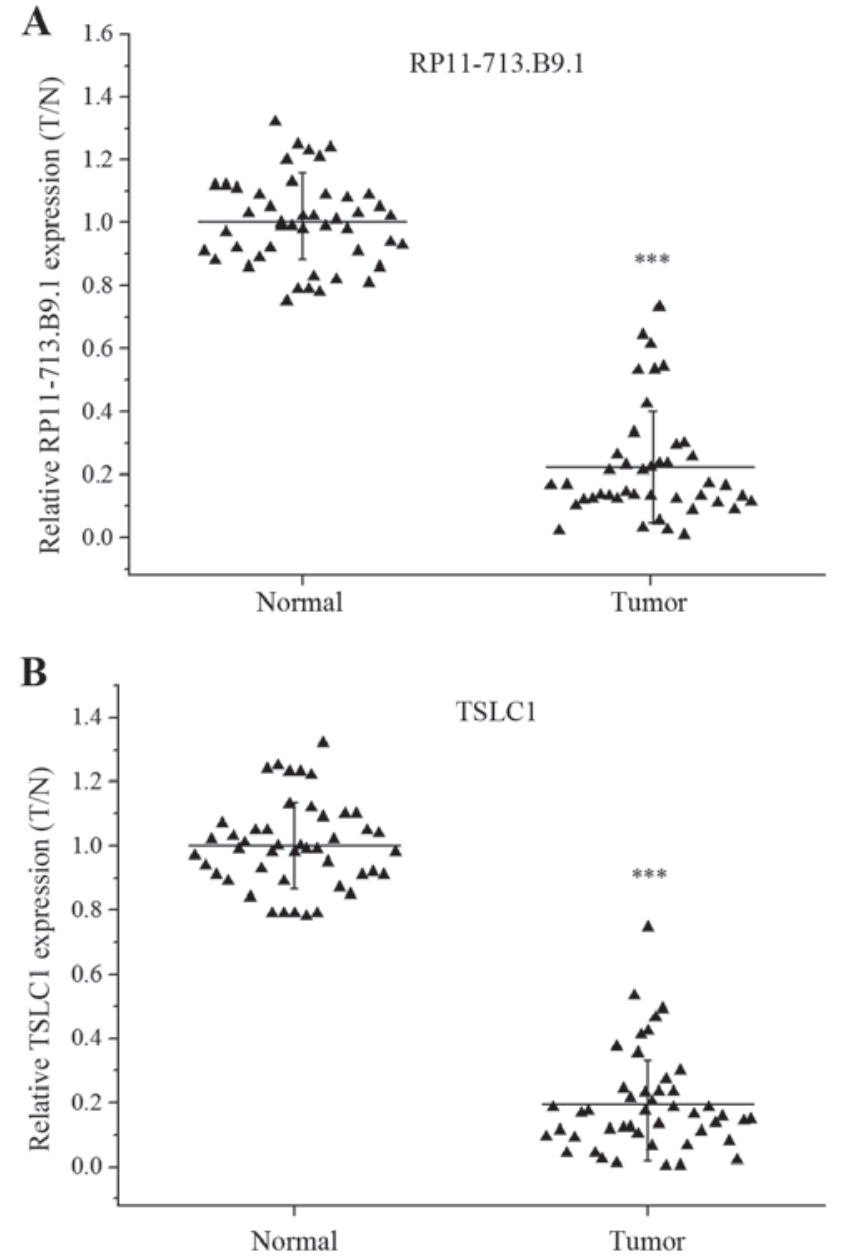

Figure 1. RP11-713B9.1 and TSLC1 expression in NSCLC compared with normal tissues. (A) RP11-713B9.1 and (B) TSLC1 expressions were significantly lower than normal tissues. ${ }^{* * * *} \mathrm{P}<0.001 ; \mathrm{T}$, tumor; $\mathrm{N}$, normal.

genes in NSCLC were also analyzed. The results confirmed that RP11-713B9.1 expression was positively correlated with tumor suppressors CYLD ( $\mathrm{R}=0.57, \mathrm{P}<0.0001$, Fig. $2 \mathrm{~B})$ and APC ( $\mathrm{R}=0.59, \mathrm{P}<0.0001$, Fig. $2 \mathrm{C})$, and negatively correlated with the oncogene BRAF $(\mathrm{R}=-0.55, \mathrm{P}<0.0001$, Fig. 2D).

Co-regulation of TSLC1 expression with RP11-713B9.1 overexpression or knockdown in NSCLC cell lines. Through Real-Time Quantitative PCR, we found that both RP11-713B9.1 and TSLC1 expression were the highest in $\mathrm{H} 460$ and the lowest in A549 among the three human NSCLC cell lines (Fig. 3A). In the overexpression experiment, when pcDNA RP11-713B9.1 was conducted and transfected into the A549, RP11-713B9.1 expression was remarkably elevated and TSLC1 expression level was also apparently up-regulated $(\mathrm{P}<0.05$, Fig. 3B), whereas in the knock-down experiment, RP11-713B9.1 expression was markedly decreased in the H460 cells after RP11-713B9.1 siRNA transfection. Quantification analysis showed that RP11-713B9.1 expression level was nearly knocked down 90\% in RP11-713B9.1 siRNA group and TSLC1 expression level was also down-regulated $(\mathrm{P}<0.05$, Fig. $3 \mathrm{C})$. It indicated that TSLC1 expression may be modulated by RP11-713B9.1 since TSLC1
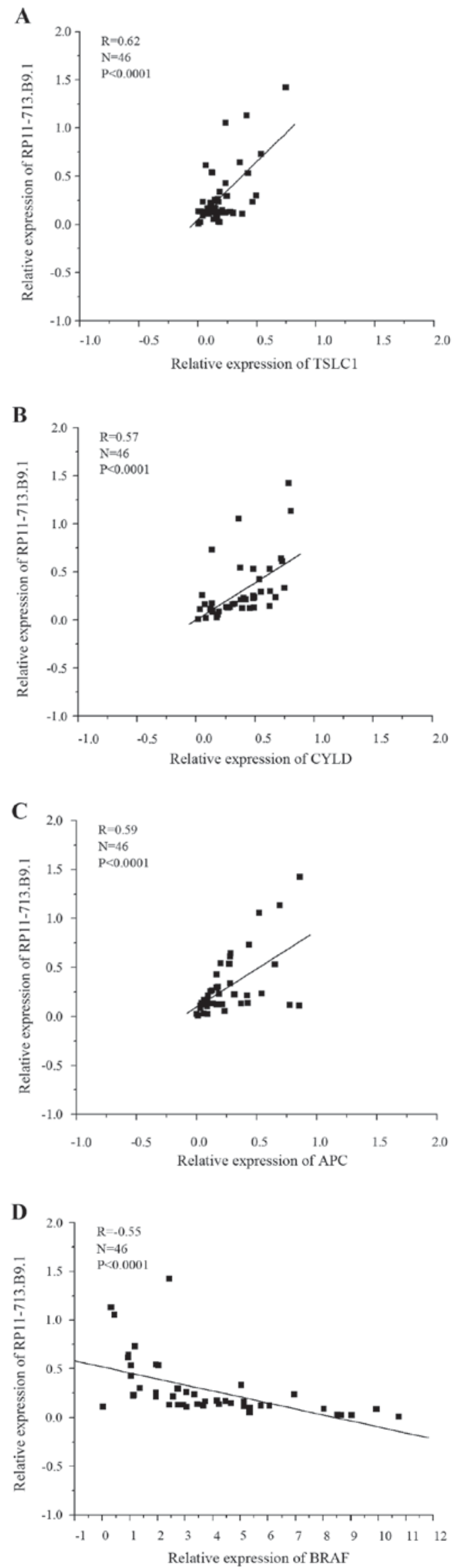

Figure 2. The correlation of RP11-713B9.1 expression with TSLC1 and other tumor related genes was assessed. RP11-713B9.1 expression was positively correlated with (A) TSLC1, (B) CYLD, (C) APC, and negatively correlated with (D) BRAF. 
A

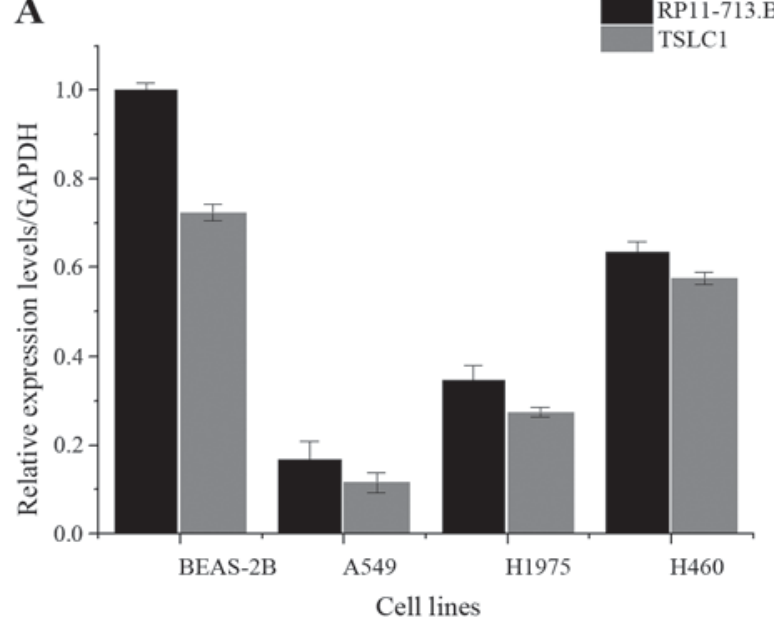

B

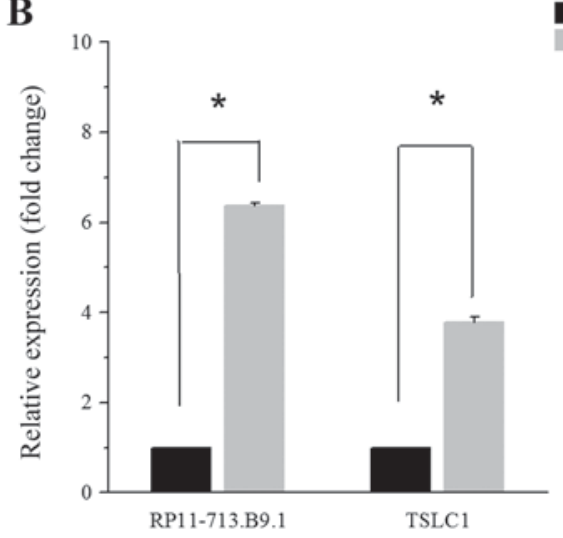

C

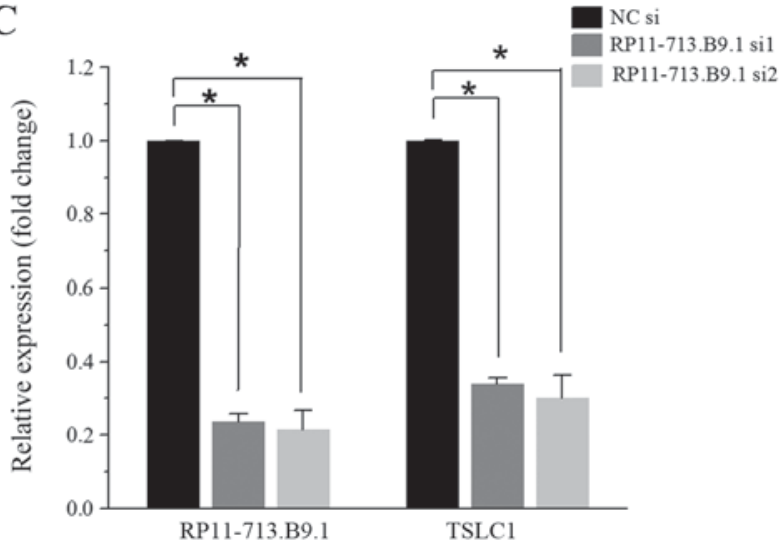

Figure 3. Co-regulation of TSLC1 expression with the RP11-713B9.1 overexpression or knockdown in the NSCLC cell lines. (A) In the three human NSCLC cell lines, the expression of RP11-713B9.1 and TSLC1 were highest in $\mathrm{H} 460$ and lowest in A549. (B) In the overexpression experiment, after pcDNA RP11-713B9.1 was conducted and transfected into the A549, RP11-713B9.1 expression and TSLC1 expression level were remarkably elevated. (C) In the knockdown experiment, RP11-713B9.1 siRNAs were transfected into the H460 cells; RP11-713B9.1 expression and TSLC1 expression level were markedly decreased. ${ }^{*} \mathrm{P}<0.05$; GAPDH, reduced glyceraldehyde-phosphate dehydrogenase; pcDNA, plasmid complementary DNA; NC, negative control; siRNA, small interfering RNA.

expression was co-regulated together with the RP11-713B9.1 in NSCLC cells.

A549 cell viability was inhibited by RP11-713B9.1 overexpression. To further analyze the role of RP11-713B9.1 in

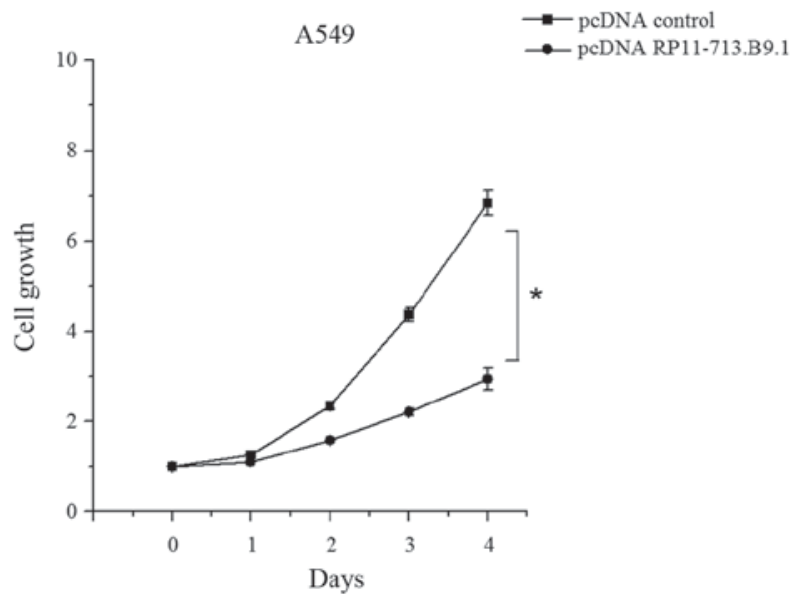

Figure 4. RP11-713B9.1 overexpression inhibited the viability of A549 cells. "P<0.05 vs. pcDNA control. pcDNA, plasmid complementary DNA.

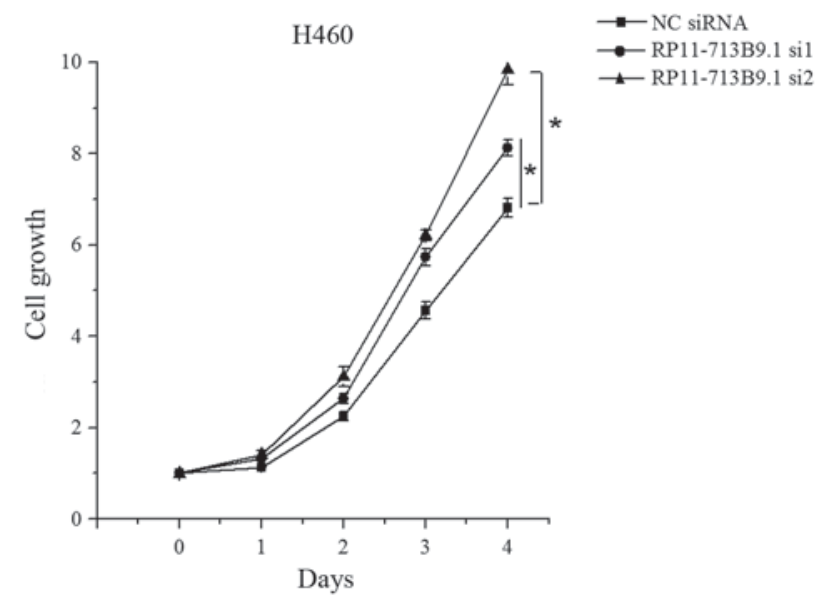

Figure 5. RP11-713B9.1 knockdown enhanced the viability of H460 cells. ${ }^{*} \mathrm{P}<0.05$ vs. NC siRNA group. NC, negative control; siRNA, small interfering RNA.

A549 cells, functional assays were conducted by transfecting pcDNA RP11-713B9.1 and pcDNA control. The results showed that the A549 cells grew evidently slower in the pcDNA RP11-713B9.1 group than those in pcDNA control group $(\mathrm{P}<0.05$, Fig. 4).

H460 cells viability was enhanced by RP11-713B9.1 knockdown. To further analyze the role of RP11-713B9.1 in H460 cells, RP11-713B9.1 knockdown experiments were conducted by transfecting RP11-713B9.1 siRNA and NC siRNA groups. The H460 cells viability was markedly promoted in the RP11-713B9.1 siRNA group when compared with the NC siRNA group $(\mathrm{P}<0.05$, Fig. 5).

\section{Discussion}

LncRNAs have been shown to exert a crucial role in tumorigenesis and contributes to a diverse of biological functions in human cancers (7-10). In recent years, aberrantly expressed lncRNAs have been reported to be implicated in regulating cell viability, migration and invasion (11-13). However, little is known about the expression and function of specified 
lncRNA in NSCLC cells. Furthermore, the biological roles of many lncRNAs in NSCLC initiation and progression are far from being well elucidated. Due to the fact that tumor metastasis is the major contributor to NSCLC related death, seeking a specific lncRNA involved in metastasis may provide novel opportunities to identify effective therapy against NSCLC.

Recent studies have identified that TSLC1 as a new tumor suppressor is often abnormally expressed in multiple cancers, including NSCLC (19-26). Intriguingly, RP11-713B9.1 is the antisense transcript of TSLC1. Despite RP11-713B9.1 as a novel lncRNA, its expression profile and functional role in NSCLC has not yet investigated, which generated our interest.

In our study, we observed that the expression of RP11-713B9.1 and TSLC1 were down-regulated in NSCLC tumors compared with matched normal tissues. Numerous protein-coding genes in the human genome have their antisense transcript partners, most of which are noncoding, just like RP11-713B9.1 and TSLC1, as a type of sense-antisense RNA duplex formation. Generally, the antisense lncRNA can modulate the sense mRNA either in a discordant or a concordant manner (31-33). For instance, HIF1 $\alpha$ together with its antisense partner aHIF has been recognized as a concordant sense-antisense RNA couple (34). Hence, we explored the correlation between RP11-713B9.1 and TSLC1 and it was demonstrated that they were positively correlated, which indicated TSLC1 may be regulated by RP11-713B9.1. Recent studies have reported some new genetic mutations that activate oncogenes, such as BRAF (27) and others that contribute to the loss of tumor suppressor function, such as CYLD, APC in NSCLC $(28,29)$. We performed the further work to investigate the correlation of RP11-713B9.1 with BRAF, APC and CYLD. Impressively, we found that the expression of RP11-713B9.1 was positively correlated with tumor suppressors APC and CYLD, and negatively correlated with the oncogene BRAF.

Moreover, we discovered that up-regulating the RP11-713B9.1 expression in low-expressing A549 cells remarkably inhibited the viability of NSCLC cells, while down-regulating the RP11-713B9.1 expression in high-expressing H460 cells showed the opposite effect. Collectively, we probably firstly found that RP11-713B9.1 was significantly down-regulated in NSCLC tissues when compared with nontumorous tissues. In addition, we also observed that RP11-713B9.1 expression was positively associated with tumor suppressors TSLC1 and CYLD, negatively correlative with oncogene BRAF. More important, loss-of and gain-of-function experiments were conducted through MTT assay, we determined that RP11-713B9.1 expression had evident effect on cell viability.

To the best of our knowledge, our data may provide the first evidence for clarifying the functional role of RP11-713B9.1 in NSCLC. These novel findings inspire us to speculate that the RP11-713B9.1 may serve as a potential biomarker and therapeutic target for NSCLC treatment in the future. Further analyses of additional clinical samples of NSCLC patients are needed as only a fraction of patient samples were analyzed in our study because of the difficulty in collection. In addition, more functional experiments like cell proliferation, migration, invasion and apoptosis will be performed to further investigate the functional roles of RP11-713B9.1 in NSCLC as we are aware that the functional experiments in our current study may not be sufficient due to the limited time. Analyzing and exploring the molecular mechanism of RP11-713B9.1 in NSCLC will be a priority for all on the basis of this paper. Above mentioned future research directions will underscore the significance of this study in near future, which will provide new insight into the effective therapy for NSCLC.

In summary, we discovered that RP11-713B9.1 and TSLC1 were down-regulated in NSCLC tissues. Meanwhile, we found that RP11-713B9.1 was positively correlated with tumor suppressors TSLC1, CYLD and APC, negatively correlated with oncogene BRAF gene. Further MTT assay showed that RP11-713B9.1 overexpression inhibited cell viability while knockdown showed the opposite effect. The data provides a new insight into the expression and functional role of RP11-713B9.1 in NSCLC. Taken together, we propose that the RP11-713B9.1 may play a crucial role in the occurrence and progression of NSCLC and has the potential to be a promising diagnostic biomarker, novel prognostic factor and therapeutic target for NSCLC in future treatment.

\section{Acknowledgements}

The authors would like to thank their colleagues for their insight and technical support.

\section{References}

1. Xu YJ, Du Y and Fan Y: Long noncoding RNAs in lung cancer: What we know in 2015. Clin Transl Oncol 18: 660-665, 2016

2. Siegel R, Ma J, Zou Z and Jemal A: Cancer statistics. CA Cancer J Clin 64: 9-29, 2014.

3. Zhang HH, Pang M, Dong W, Xin JX, Li YJ, Zhang ZC, Yu L, Wang PY, Li BS and Xie SY: miR-511 induces the apoptosis of radioresistant lung adenocarcinoma cells by triggering BAX. Oncol Rep 31: 1473-1479, 2014.

4. Ma Y, Li X, Cheng S, Wei W and Li Y: MicroRNA-106a confers cisplatin resistance in non-small cell lung cancer A549 cells by targeting adenosine triphosphatase-binding cassette A1. Mol Med Rep 11: 625-32, 2015.

5. Saito M, Shiraishi K, Matsumoto K, Schetter AJ, Ogata-Kawata H, Tsuchiya N, Kunitoh H, Nokihara H, Watanabe S, Tsuta K, et al: A three-microRNA signature predicts responses to platinum-based doublet chemotherapy in patients with lung adenocarcinoma. Clin Cancer Res 20: 4784-4793, 2014.

6. Rinn JL and Chang HY: Genome regulation by long noncoding RNAs. Annu Rev Biochem 81: 145-166, 2012.

7. Guttman M, Amit I, Garber M, French C, Lin MF, Feldser D, Huarte M, Zuk O, Carey BW, Cassady JP: Chromatin signature reveals over a thousand highly conserved large non-coding RNAs in mammals. Nature 458: 223-227, 2009.

8. Huarte M and Rinn JL: Large non-coding RNAs: Missing links in cancer? Hum Mol Genet 19: R152-R161, 2010.

9. Spizzo R, Almeida MI, Colombatti A and Calin GA: Long non-coding RNAs and cancer: A new frontier of translational research? Oncogene 31: 4577-4587, 2012.

10. Tsai MC, Spitale RC and Chang HY: Long intergenic noncoding RNAs: New links in cancer progression. Cancer Res 71: 3-7, 2011.

11. Wang F, Yuan JH, Wang SB, Yang F, Yuan SX, Ye C, Yang N, Zhou WP, Li WL, Li W and Sun SH: Oncofetal long noncoding RNA PVT1 promotes proliferation and stem cell-like property of hepatocellular carcinoma cells by stabilizing NOP2 Hepatology 60: 1278-1290, 2014.

12. Yuan JH, Yang F, Wang F, Ma JZ, Guo YJ, Tao QF, Liu F, Pan W, Wang TT, Zhou CC, et al: A Long Noncoding RNA Activated by TGF- $\beta$ Promotes the Invasion-Metastasis Cascade in Hepatocellular Carcinoma. Cancer Cell 25: 666-681, 2014. 
13. Zhou X, Liu S, Cai G, Kong L, Zhang T, Ren Y, Wu Y, Mei M, Zhang $L$ and Wang $X$ : Long non coding RNA MALAT1 promotes tumor growth and metastasis by inducing epithelial-mesenchymal transition in oral squamous cell carcinoma. Sci Rep 5: 15972, 2015.

14. Sun M, Liu XH, Wang KM, Nie FQ, Kong R, Yang JS, Xia R, $\mathrm{Xu}$ TP, Jin FY, Liu ZJ, et al: Downregulation of BRAF activated non-coding RNA is associated with poor prognosis for non-small cell lung cancer and promotes metastasis by affecting epithelial-mesenchymal transition. Mol Cancer 13: 68, 2014.

15. Gutschner T, Hämmerle M, Eissmann M, Hsu J, Kim Y, Hung G, Revenko A, Arun G, Stentrup M, Gross M, et al: The noncoding RNA MALAT1 is a critical regulator of the metastasis phenotype of lung cancer cells. Cancer Res 73: 1180-1189, 2013.

16. Nowacki S, Skowron M, Oberthuer A, Fagin A, Voth H, Brors B, Westermann F, Eggert A, Hero B, Berthold F and Fischer M: Expression of the tumour suppressor gene CADM1 is associated with favourable outcome and inhibits cell survival in neuroblastoma. Oncogene 27: 3329-3338, 2008.

17. Fischer M, Oberthuer A, Brors B, Kahlert Y, Skowron M, Voth H, Westermann F, Eggert A, Hero B, Berthold F and Fischer M: Differential expression of neuronal genes defines subtypes of disseminated neuroblastoma with favorable and unfavorable outcome. Clin Cancer Res 12: 5118-5128, 2006.

18. Watabe K, Ito A, Koma YI and Kitamura Y: IGSF4: A new intercellular adhesion molecule that is called by three names, TSLC1, SgIGSF and SynCAM, by virtue of its diverse function. Histol Histopathol 18: 1321-1329, 2003.

19. Lung HL, Cheung AK, Xie D, Cheng Y, Kwong FM, Murakami Y, Guan XY, Sham JS, Chua D, Protopopov AI, et al: TSLC1 is a tumor suppressor gene associated with metastasis in nasopharyngeal carcinoma. Cancer Res 66: 9385-9392, 2006.

20. Heller G, Geradts J, Ziegler B, Newsham I, Filipits M, Markis-Ritzinger EM, Kandioler D, Berger W, Stiglbauer W, Depisch D, et al: Downregulation of TSLC1 and DAL-1 expression occurs frequently in breast cancer. Breast Cancer Res Treat 103: 283-291, 2007.

21. Honda T, Tamura G, Waki T, Jin Z, Sato K, Motoyama T, Kawata S, Kimura W, Nishizuka S and Murakami Y: Hypermethylation of the TSLC1 gene promoter in primary gastric cancers and gastric cancer cell lines. Jpn J Cancer Res 93: 857-860, 2002.

22. Jansen M, Fukushima N, Rosty C, Walter K, Altink R, Heek TV, Heek TV, Hruban R, Offerhaus JG and Goggins M: Aberrant methylation of the 5-CpG island of TSLC1 is common in pancreatic ductal adenocarcinoma and is first manifest in high-grade PanlNs. Cancer Biol Ther 1: 293-296, 2002.
23. Chen K, Wang G, Peng L, Liu S, Fu X, Zhou Y, Yu H, Li A, Li J, Zhang S, et al: CADM1/TSLC1 inactivation by promoter hypermethylation is a frequent event in colorectal carcinogenesis and correlates with late stages of the disease. Int J Cancer 128: 266-273, 2011.

24. Yang YX, Yang AH, Yang ZJ, Wang ZR and Xia XH: Involvement of tumor suppressor in lung cancer 1 gene expression in cervical carcinogenesis. Int J Gynecol Cancer 16: 1868-1872, 2006.

25. Williams YN, Masuda M, Sakurai-Yageta M, Maruyama T, Shibuya $M$ and Murakami Y: Cell adhesion and prostate tumor-suppressor activity of TSLL2/IGSF4C, an immunoglobulin superfamily molecule homologous to TSLC1/IGSF4. Oncogene 25: 1446-1453, 2006.

26. Kuramochi M, Fukuhara H, Nobukuni T, Kanbe T, Maruyama T, Ghosh HP, Pletcher M, Isomura M, Onizuka M, Kitamura T, et al: TSLC1 is a tumor suppressor gene in human non-small cell lung cancer. Nat Genet 27: 427-430, 2001.

27. Nguyen-Ngoc T, Bouchaab H, Adjei AA and Peters S: BRAF alterations as therapeutic targets in non-small-cell lung cancer. J Thorac Oncol 10: 1396-1403, 2015.

28. Deng LL, Shao YX, Lv HF, Deng HB and Lv FZ: Over-expressing CYLD augments antitumor activity of TRAIL by inhibiting the $\mathrm{NF}-\kappa \mathrm{B}$ survival signaling in lung cancer cells. Neoplasma 59: $18-29,2012$.

29. Guo S, Tan L, Pu WL, Wu J, Xu K, Wu J, Li Q, Ma Y, Xu J, Jin L and Wang J: Quantitative assessment of the diagnostic role of APC promoter methylation in non-small cell lung cancer. Clin Epigenetics 6: 5, 2014

30. Qin X, Yao J, Geng P, Fu X, Xue J and Zhang Z: LncRNA TSLC1-AS1 is a novel tumor suppressor of glioma. Int J Clin Exp Pathol 7: 3065-3072, 2014.

31. Wahlestedt C: Natural antisense and noncoding RNA transcripts as potential drug targets. Drug Discov Today 11: 503-508, 2006

32. Faghihi MA and Wahlestedt C: Regulatory roles of natural antisense transcripts. Nat Rev Mol Cell Biol 1: 637-643, 2009.

33. Yelin R, Dahary D, Sorek R, Levanon EY, Goldstein O, Shoshan A, Diber A, Biton S, Tamir Y, Khosravi R, et al: Widespread occurrence of antisense transcription in the human genome. Nat Biotechnol 21: 379-386, 2003.

34. Thrash-Bingham CA and Tartof KD: aHIF: A natural antisense transcript overexpressed in human renal cancer and during hypoxia. J Natl Cancer Inst 91: 143-151, 1999. 